\title{
CERTEZA ANIMAL: ¿UN TERCER WITTGENSTEIN?
}

\author{
ANIMAL CERTAINTY: A THIRD WITTGENSTEIN?
}

\section{CRISTIÁN SANTIBÁÑEZ YÁÑEZ}

\section{RESUMEN}

Discuto en este trabajo algunas de las interpretaciones que en este último tiempo se han hecho de Sobre la certeza de Ludwig Wittgenstein con el objeto de distinguir hasta qué punto se puede hablar de un tercer Wittgenstein. Los temas discutidos son: la novedad e importancia atribuida a esta obra; la supuesta subversión de la perspectiva tradicional sobre las creencias básicas; y el 'nuevo' anti-escepticismo que superaría el sentido común de Moore como respuesta al escepticismo radical. La discusión intenta ser de ayuda para diferenciar estas claves que, como otras de Wittgenstein, pueden tener repercusión en distintas áreas del saber.

Palabras clave: Proposiciones bisagras, escepticismo, anti-escepticismo, estructura de razones, Wittgenstein.

\section{ABSTRACT}

In this paper some of the latest interpretations of Ludwig Wittgenstein's On Certainty are analysed in order to distinguish to what extent it is possible to talk about a 'third' Wittgenstein. The topics discussed are: the novelty and importance attributed to On Certainty; the subversion of the traditional perspective about basic beliefs; and the 'new' anti-scepticism which exceeds Moore's common sense as an answer to radical scepticism. Furthermore, this discussion tries to offer some support in differentiating

\footnotetext{
${ }^{1}$ Este trabajo es parte de mi investigación post-doctoral en el marco del Proyecto FONDECYT 3090017 "Principio de Relevancia: Dimensiones Cognitiva y Argumentativa".

${ }^{2}$ Centro de Estudios de la Argumentación y el Razonamiento Universidad Diego Portales. Santiago, Chile. E-mail: Cristian.santibanez@udp.cl
} 
these keys that, like others of Wittgenstein, could have consequences in different fields of knowledge.

Keywords: Hinge propositions, scepticism, anti-scepticism, reasons structure, Wittgenstein.

Recibido: 17.03.10. Aceptado: 25.09.10.

\section{INTRODUCCIÓN}

" E LO que no se puede hablar, hay que callar" (Tractatus LogicoPhilosophicus, $\$ 7$ ), y el lenguaje es su "uso" en el marco -del aprendizaje- de "juegos de lenguaje" (Investigaciones filosóficas, $\$ 7)$, son dos de las ideas comunes de Wittgenstein citadas en muchas áreas del conocimiento, tales como la literatura, el psicoanálisis, la lingüística, la sociología, la antropología, la psicología, por nombrar algunas, para desarrollar análisis, interpretar textos, o dar sentido a los mensajes involucrados. Como se suele apuntar, la primera idea se atribuye a un joven, o primer Wittgenstein, y el segundo tramado de nociones se atribuye a un tardío o segundo Wittgenstein.

Para aquellos que aún sienten que esta parcelación es antojadiza, la noticia del "descubrimiento" de un tercer Wittgenstein sonará, entonces, ya un despropósito. Sin embargo, actualmente los estudiosos y críticos dedicados a la obra wittgensteiniana más respetados por la academia internacional, hablan de la existencia de un tercer Wittgenstein a partir de lo que el autor entrega en Sobre la certeza.

"Si sabes que aquí hay una mano, te concederemos todo lo demás". Este es, como se sabe, el enunciado con el que Wittgenstein inicia Sobre la certeza (en adelante OC) ${ }^{3}$. Como en tantas ocasiones en sus escritos, afirmaciones de este tipo nos han causado perplejidad, tanto por su brevedad como por su aparente simplicidad. Las notas que dan vida a OC mantuvieron ocupado a Wittgenstein hasta dos días antes de su muerte. Este es un dato que parece anecdótico, pero en realidad, como se discutirá luego, se alza como sustancial para parte de la crítica especializada que ha estado trabajando últimamente en esta obra.

Considerando tanto algunos aspectos que pueden ser vistos como globales de un proyecto filosófico en Wittgenstein, como ciertos elementos de

\footnotetext{
${ }^{3}$ Se utilizará la nomenclatura "OC" para referirnos a Sobre la certeza, siguiendo la convención entre los especialistas de habla inglesa.
} 
comparación entre OC y, principalmente, el Tractatus y las Investigaciones, en este trabajo analizo y discuto lo que distingue a OC como un trabajo especial del legado del filosofo austriaco, a partir de los siguientes ejes: a) la importancia atribuida a OC en virtud de lo que parte de la crítica reconoce como lo más sustancial de esta obra; b) la noción de "proposiciones bisagras" que, precisamente, la crítica coincide en señalar que se trata de la propuesta fundamental de OC; $y$ c) la perspectiva y alcances que se le achacan a Wittgenstein en OC respecto del escepticismo y anti-escepticismo. Las conclusiones de este trabajo retoman algunas interrogantes que no se despejan del todo en las lecturas cruzadas entre los críticos y sugieren dimensiones que podrían ser parte de una agenda de investigación e interpretación de OC.

\section{DE LA IMPORTANCIA ATRIBUIDA A OC}

Tal como lo ha señalado generosamente Malcolm $(1986)^{4}$, en OC el lector se enfrenta a ideas de Wittgenstein genuinamente nuevas, que difícilmente pueden ser indicadas como repetición de algún pensamiento anterior. Esto no quiere decir, por cierto, que $\mathrm{OC}$ no forme parte de un proyecto filosófico más amplio, o que incluso se encuentre en relación crítica con perspectivas anteriores. Por el contrario, y como se irá observando en las líneas que siguen, OC es un trabajo de vínculo y distanciamiento de ciertas convicciones que Wittgenstein tuvo en otros momentos.

Comenzaré por señalar, por lo pronto, que tres razones de las que esgrime Avrum Stroll (2007) me importan respecto de la relevancia que se le otorga a $\mathrm{OC}^{5}$. La primera razón que rescato es que OC expone un tipo de fundacionalismo no tratado previamente; segundo, una visión renovada del modo en que conocemos y formamos certezas; y tercero, provee de una distinción, no apuntada en la literatura filosófica anterior, entre certeza subjetiva y certeza objetiva.

\footnotetext{
${ }^{4}$ Sus elogiosas palabras son las siguientes: "Wittgenstein's last notebooks, published under the title On Certainty, were written in the final year and a half of his life. They are rough notes, completely unrevised. They are his discussions with himself, with no anticipation of publication. ... Many readers find the whole thing bewildering. But these notes reward hard study. Not only are there individual comments of great beauty, but also lines of thought emerge that are not to be found elsewhere in Wittgenstein's writings” (Malcolm 1986, p. 201).

${ }^{5}$ En un juicio un tanto extremista, Stroll (2007, p. 33) sostiene de entrada, y sin atenuación, que las notas que se recopilan bajo OC dan vida al libro más importante en la filosofía occidental desde La crítica de la razón pura de Kant.
} 
Respecto del fundacionalismo, Stroll (2007) enfatiza que la idea de que los juegos de lenguaje descansan en una base fundada, o fundamento, está completamente ausente en las Investigaciones, por lo que con OC nos encontramos frente a una etapa totalmente distinta y nueva de Wittgenstein. Uno de los núcleos para Stroll en OC es que los fundamentos de los juegos de lenguaje están fuera de los mismos, pero los mantienen. Así, la idea de que Wittgenstein es un "fundacionalista" en esta etapa de su vida, es altamente controversial ${ }^{6}$. Pero un dato estadístico es lo suficientemente ilustrativo para comenzar a disipar cualquier duda: de las 676 entradas, o parágrafos, que OC contiene, en más del $10 \%$ de ellas aparece explícitamente la palabra "fundamento", o "base", para mostrar el contraste existente entre la idea de juegos de lenguaje y lo que se encuentra, metafórica o literalmente para Wittgenstein, por debajo de ellos y los soportan. Ejemplo cualitativo: " $\$ 205$. Si lo verdadero es lo que tiene fundamentos, el fundamento no es verdadero, ni tampoco falso."

La segunda razón que esgrime Stroll (2007), para sostener que OC es una compilación única, se vincula con lo que para este autor es una nueva epistemología en y a través de Wittgenstein, y que tiene como blanco la distinción entre "certeza" y "saber". Mientras el fundamento funciona como certeza objetiva para la justificación, el "saber" funciona como y en enunciados de lo empírico. Muestras claras de esta distinción son los parágrafos 116 y $341-343$, pero sin duda alguna en $\$ 308$, en los que se disipa cualquier duda respecto de que "saber" y "certeza" apuntan a dos dimensiones distintas en los procesos de justificación. De acuerdo a Stroll (2007), para Wittgenstein "saber" y "certeza" (o seguridad) no son estados mentales, sino comportamientos distintos. Mientras la primera categoría fluye para proposiciones mayormente empíricas en ciertos contextos, la segunda está vinculada a contextos en que el error no es posible, e incluso, a proposiciones que no se debieran enunciar, como por ejemplo "Se qué tengo un cuerpo". Lo ilustrativo para Stroll (2007, p. 37) está en $\$ 195$ : “Si creo que estoy sentado en mi habitación cuando no lo estoy, entonces no debiera decirse que he cometido un error". Un error, nos recuerda Wittgenstein en $\$ 156$, es un equívoco que cabe en los patrones de lo normal en las actividades humanas, como en el caso de un cálculo errado. Es un juicio erróneo el que se hace en conformidad a lo permitido. Para Stroll (2007) esta forma de exponer

${ }^{6}$ Para Williams (2007), por ejemplo, Wittgenstein no es fundacionalista en OC, o al menos no un fundacionalista tradicional, ya que los 'juicios marcos' de Wittgenstein son extremadamente heterogéneos entre sí y no obedecen a ningún tipo de esencia o base común que pueda reconocerse como fundamento. 
la diferencia entre tales categorías es, además, la posición fundamental de Wittgenstein contra el escepticismo, en tanto que certeza objetiva ("Se que tengo un cuerpo") no se "dice", pero se enuncia con fines mostrativos. Por su parte, la certeza subjetiva, en lectura de Stroll, está destinada a medirse en base al juego del error en el marco de nuestros intentos por convencer.

La distinción entre "certeza objetiva" y "certeza subjetiva" es la tercera razón que Stroll (2007, p. 36) formula para otorgarle una importancia decisiva a OC en el marco de la inauguración de una nueva epistemología. Como se ha señalado, la "certeza objetiva" se identifica con casos donde un error no es posible, a menos claro, como señala Stroll (2007, p. 38), uno quiera poner en duda la herencia de un mundo o la participación en una comunidad; la "certeza subjetiva", en cambio, se vincula con el ejercicio efectivo de la certeza objetiva en contextos donde el señalamiento esté en función de convencer. Dicho de otra forma, la certeza subjetiva es la formulación de la certeza objetiva por parte de un individuo con fines de evidencia, aun cuando para Wittgenstein esto es, si no innecesario, al menos redundante.

Según Stroll (2007), en OC se despliega la brillantez de Wittgenstein en lo concerniente a su entendimiento, más profundo, del problema del escepticismo, siendo éste menos un desafío a la existencia del conocimiento que a la existencia de la certeza. Las certezas están, con la metáfora de un objeto en el tiempo, más allá del saber y el conocer, sin ellas estos últimos son categorías sin sentido.

\section{CERTEZA ANIMAL Y PROPOSICIONES BISAGRAS ${ }^{7}$}

Tal como lo reconoce el propio Stroll (2007, p. 34), se le debe a MoyalSharrock (2004) el que se hable de "el tercer Wittgenstein" sobre la base de lo que se nos entrega en OC. Para Moyal-Sharrock (2007) esta nueva nomenclatura sobre Wittgenstein se justifica porque en OC el autor, en progresión respecto de las Investigaciones (incluso respecto del Tractatus, de acuerdo a Rhees (2003)), se ocupa de las formas primitivas del lenguaje $y$, por tanto, de los "fundamentos" de los juegos de lenguaje. Estas formas primitivas son, como señala el propio Wittgenstein $(\mathrm{CV} \$ 31)^{8}$, reaccio-

\footnotetext{
${ }^{7}$ Se ha traducido literalmente "hinge propositions" por "proposiciones bisagras" para conservar la riqueza y coherencia metafórica con la que Wittgenstein explica este tipo de proposiciones. La metáfora bien queda de manifiesta en OC $\$ 341$.

${ }^{8}$ Con la abreviación CV me refiero al texto Culture and Value de Wittgenstein.
} 
nes, comportamientos, actos físicos. De modo que, y grosso modo, si en el Tractatus estamos en presencia del primer Wittgenstein (donde el lenguaje se estudia como realidad lógica), y en las Investigaciones frente al segundo Wittgenstein (donde el lenguaje y los problemas filosóficos se estudian a partir de parecidos de familia y la comprensión y seguimiento de reglas), en OC estamos, entonces, frente al tercer Wittgenstein (donde el lenguaje es tributario de una realidad biológica básica y un comportamiento en comunidad irreducible).

Para Rhees (2003) esta división es antojadiza. Sin dejar de reconocer la progresión existente en Wittgenstein respecto de los problemas que éste trata, Rhees sostiene que la preocupación de Wittgenstein, en la mayor parte de su producción, fue el problema del lenguaje, por lo que mal hay etapas sino simplemente distintos énfasis y problemas específicos en los que éste se manifiesta (como por ejemplo: en la noción de color, en el lenguaje matemático, en el problema religioso, en el problema lógico, e incluso en el problema discursivo).

Sin reparar en este contra-argumento, Moyal-Sharrock $(2005,2007)$ ha insistido en que lo principal en OC se encuentra en la necesidad de distinguir entre "certeza objetiva" y "certidumbres objetivas". La primera se expresa, justamente, como certeza animal (OC $\$ 359$ ), como un tipo de seguridad que no tiene, y no le cabe, justificación, que no posee razones. Por ejemplo, "aquí tengo una mano y aquí la otra", "estoy sentado", "tengo un cuerpo", son proposiciones todas que no tienen mayor justificación que la confianza ciega, no-reflexiva, de que contamos con eso. A pesar de que "no conocemos" tales proposiciones, actuamos sobre ellas. Por tanto, a juicio de Moyal-Sharrock (2007, p. 86; véase también Pritchard, por aparecer) estas proposiciones son fundacionales.

Para la autora, estas proposiciones son fundacionales en dos sentidos: en tanto categoría doxática, por un lado, y en tanto actitud doxática, por otro. Mientras la primera se refiere a aquellas creencias, dentro de nuestros(s) sistema(s) de creencias, que "actúan como reglas de gramática", la segunda se refiere a aquella manifestación no proposicional y sus objetos "pertenecen a la gramática". La certeza objetiva en tanto categoría doxática se observa en OC cuando Wittgenstein habla de que con ciertas proposiciones "estamos en la base", "hemos alcanzado la piedra angular". En una nomenclatura más tradicional, estas metáforas también se refieren a la noción alemana “Weltbild" (OC $\$ 94, \$ 162$ ). Todos nuestros juegos de lenguaje, todas nuestras acciones y pensamientos están basados en este fondo (OC $\$ 253$, $\$$ $492, \S 97, \S 162, \S 248, \S 211, \S 94, \S 151, \S 96, \S 144, \S 655, \S 403, \S 103)$. 
También cabe hablar de "marco de referencia" (OC $\$ 83$ ). El concepto que se ha impuesto, no obstante, es el de "proposiciones bisagras", que son de tal tipo y envergadura que han evolucionado hacia convicciones, pero que también, y lo fundamental para Wittgenstein, se convierten en un método de duda e investigación.

La certeza objetiva en tanto actitud doxática se manifiesta, de acuerdo a la lectura de Moyal-Sharrock $(2005,2007)$, como un tipo de confianza ciega en lo que hacemos y mostramos al decir y al actuar, y su objeto es parte de la gramática (OC $\$ 153$ ), como cuando son objetos de experiencia (OC $\$$ 337): "Aquí hay una mano". Estas proposiciones funcionan como actitudes, tal como las reacciones, es decir, como normas de descripción.

Respecto de las "certidumbres objetivas", Moyal-Sharrock las define como los componentes específicos de las redes fundacionales que se mantienen unidas (tal como señala Wittgenstein en OC $\$ 279, \S 234, \S 141$ ). Pero la asimilación de ellas, y esto es lo sugerente de la posición de MoyalSharrock, no es ni proposicional, ni empírica, ni epistémica. Las certidumbres objetivas no son trascendentales, sino que son subjetivas, pero subjetivas en el sentido de que son indesmentibles tanto para mí como para cualquier otro individuo. La certeza objetiva se disecciona en certidumbres objetivas, y éstas a su vez, dan forma, en vida corriente, de lo que hacemos en la actividad mundana (OC $\$ 204$ ).

Romper con la perspectiva tradicional de construir nuestra relación con la certeza como materia de saber es la importancia que le atribuye MoyalSharrock a OC, pues no se trata de que sepamos o conozcamos la certeza, sino de lo que hacemos y mostramos cuando actuamos con y sobre ella. Es un tipo de seguridad que no tiene precursor en el pensamiento, es instantánea... excepto cuando filosofamos (OC $\$ 407)$.

Creo que una consecuencia directa de esta forma de ver las cosas es la filosofía pragmática y naturalista sobre los fenómenos intencionales y el acto de dar razones de Searle (2001). A este tipo de certidumbre Searle (2001) le denomina "compromiso". Para Searle "compromiso" es el set de habilidades y estados que, no siendo ellos mismo intencionales, hacen posible contenidos intencionales". A esto Wittgenstein le llama "algo animal" (OC $\$ 510)$, en tanto rudimento básico, elemental, primigenio sobre el que se construyen diálogos naturales. Se debe insistir, se trata de una confianza ciega (OC

\footnotetext{
${ }^{9}$ Searle (2002) también utiliza el concepto "background" para referirse a la misma idea, esto es, un set de habilidades y conocimiento en los agentes que permite no sólo el funcionamiento del lenguaje sino además lo que Searle llama "intencionalidad compartida”.
} 
$\$ 150$ ), una creencia no razonada, un tipo de actitud que compartimos con los neonatos y la fauna cercana a nosotros. Esta confianza no se experimenta qua confianza, sino en la inexistencia de desconfianza. No requieren atención cognitiva, y operan por "default"; es decir, a menos que exista una excepción, ellas fluyen sin más. Son no-reflexivas. Prestar atención a una certeza bisagra es signo inequívoco que algo vital se está poniendo en duda o está funcionando mal. Cuando sostenemos "Sé que esto es una mano" nos mostramos como practicando un caso raro, donde tal proposición no está funcionando como bisagra, sino como descripción o conclusión. Cuando Moore sostiene "Aquí hay una mano" no está demostrando nada, sino que mostrando algo, algo que nunca estuvo escondido. La certeza animal no sólo es ciega, sino además silente.

Las proposiciones bisagras, como "Tengo un cuerpo", que no las digo sino que sólo las hablo en contadísimas oportunidades ${ }^{10}$, sobre todo cuando estamos en contextos muy intelectualizados, son reglas gramaticales en el sentido de que condicionan el uso de nuestras palabras y oraciones. En tanto ocurrencias o manifestaciones, las proposiciones bisagras son inefables.

Moyal-Sharrock (2007) propone, en síntesis, que las proposiciones bisagras son: indudables (duda y error son lógicamente carentes de sentido en ellas); fundacionales (no resultan de ninguna justificación); no-empíricas (no son derivadas de los sentidos); gramaticales (son reglas de la gramática); inefables (no se pueden decir); y se observan en acción (ellas se muestran a sí mismas en la forma en que actuamos). Otras taxonomías de Moyal-Sharrock (2007, p. 102), que no se presentan sin embargo con total detalle, aparecen bajo títulos como: bisagras lingüísticas $(2+2=4$, o "Las palabras que componen esta oración están en Español”, que son adquiridas); bisagras personales ("Por meses he tenido la dirección $\mathrm{xxx");} \mathrm{bisagras}$ locales ("Hay una isla, Isla de Pascua"); bisagras universales ("La tierra existe", que son naturales e instintivas).

En OC Wittgenstein insiste en que nuestra certeza objetiva es práctica, de la misma forma en que nuestra adherencia a una regla de pensamiento puede tener sentido sólo si se manifiesta ella misma en nuestros actos. Un rechazo verbal a una ley de pensamiento no es efectivo en términos lógicos. La frase "No podemos dudar de todo" articula una ley de pensamiento hu-

\footnotetext{
${ }^{10}$ Moyal-Sharrock (2007, p. 91) distingue entre decir y hablar las proposiciones bisagras, pues en tanto reglas gramáticas no se "dicen", pero pueden ser "enunciadas", como por ejemplo cuando tienen un objetivo descriptivo al enseñar a un niño una lengua.
} 
mana, expresa una forma de actuar y pensar normales en nuestro mundo. Cualquier rechazo no tiene ninguna resonancia práctica en nuestras vidas. Si lo hacemos, somos declarados dementes.

\section{ANTI-ESCEPTICISMO Y ESTRUCTURA DE LAS RAZONES}

Respecto de las proposiciones bisagras, el anti-escepticismo y la crítica a Moore contenidos en OC, Duncan Pritchard (2007; y por aparecer) ofrece elementos diferentes para el análisis. Primero que nada, Pritchard señala que la idea de proposiciones bisagras sirve para distinguir la forma de la estructura de dar razones, incorporando la exclusión de la total y general evaluación epistémica esencial al argumento escéptico: hay cosas respecto de las que no cabe dudar. Sin embargo, a Pritchard no le interesa tanto esto como un segundo argumento más implícito en la posición de Wittgenstein sobre el escepticismo. Este segundo argumento va como sigue: de acuerdo a Pritchard, para Wittgenstein el escéptico está totalmente divorciado del lenguaje ordinario, y las consecuencias dialécticas de este divorcio van más allá del problema de la relevancia en el uso de proposiciones en ciertos contextos, de modo que el ataque de Moore al escéptico se construye sobre la base de una concepción errónea de la estructura de las razones, de cómo éstas se engranan. Para pretender saber de forma apropiada a través de una proposición, es esencial que uno esté disponible a ofrecer poderosas razones a su favor. Cuando sostenemos algo que es demasiado cierto, ¿qué razones podemos esgrimir para justificarlas?, si tales razones no son más poderosas que la proposición misma, no juegan su rol de justificación. El papel dialéctico en la estructura de las razones de la pretensión de saber es resolver dudas a través de razones que soporten otras, lo que no se cumple cuando tenemos las proposiciones bisagras, cuando se sostiene "Aquí tengo una mano". Dicho de otra forma, se sostiene una pretensión de saber allí donde hay un desafío contextual relevante a lo afirmado (¿Alguien duda de que tengamos manos?). Enumerar por enumerar, mostrar por mostrar, no tiene ningún rol dialéctico. Para Pritchard este argumento de Wittgenstein se observa con claridad en OC $\$ 125$.

De acuerdo a Pritchard (por aparecer), Wittgenstein quiere erradicar el hábito de abusar de la concepción de duda, de las expresiones de duda, erradicar la idea de que ellas son "libres", ya que, en realidad, ellas no pueden entrar a un contexto conversacional sin una motivación, de otra forma son incoherentes (OC $\$ 122-123)$. Las proposiciones que más conocemos 
no pueden ser declaradas como conocidas, o dudadas, porque tales proposiciones, las bisagras, están fuera de la ruta de viaje de la investigación.

Pritchard (2007), sin embargo, se esfuerza por hacer notar que la clase de indudabilidad que Wittgenstein promueve no es la clásica promovida por la epistemología tradicional. Cuando Descartes trata al cogito como indudable, y así fundacional, tiene en mente una concepción de indudabilidad donde dudar de la proposición en cuestión ipso facto asegura su verdad. La indudabilidad del cogito es así una indicación de su verdad. En Wittgenstein no se encuentra esta posición, sino simplemente el hecho de que sobre ciertas materias no cabe dudar porque no hay soporte racional, nada corre a su auxilio en el ámbito de lo decible.

No obstante, este mismo argumento va en contra del anti-escéptico, en el entendido de que Wittgenstein reclama una arquitectura de la estructura de las razones en la que las proposiciones bisagras son su corazón, y no es sólo una limitación práctica a nuestros comportamientos el que ciertas proposiciones estén exentas de evaluación epistémica, sino que están aparte de la lógica misma de la evaluación epistémica. Así, no podemos ofrecer razones para los equívocos, sino que debemos buscar, si cabe, sus causas.

Convengamos, por último, que Pritchard (2007) coincide con MoyalSharrock $(2007,2005)$ en que las proposiciones bisagras expresan normas más que hechos, que deben observarse como “algo animal” (OC $\$ 358$ 359), y que no tienen naturaleza epistémica.

\section{CONCLUSIONES}

Como se ha intentado dejar constancia, parte importante de la crítica especializada en Wittgenstein ha visto con renovado interés las notas de $\mathrm{OC}^{11}$. Por las razones y perspectivas discutidas en el cuerpo de este trabajo, queda de manifiesto que OC abre una nueva veta de análisis en la obra de Wittgenstein con amplias repercusiones. Esto, que parece evidente, no es del todo compartido o visualizado por comentaristas tradicionales de Wittgenstein. Por ejemplo, Soames (2003) en su revisión y proyección de Wittgenstein nada comenta sobre la importancia de OC. En una posición

${ }^{11}$ En Stroll, sin embargo, la preocupación por el problema de la "certeza" en Moore y Wittgenstein se puede observar en publicaciones más tempranas a esta última ola de literatura en torno al tema; véase Stroll (1994). 
intermedia se encuentra Von Wright (1982), para quien OC es nudo crítico aun incompleto. El otro extremo está representado, entre otros, por Rhees (2003), para quien OC es el trabajo más elocuente en el proyecto filosófico de Wittgenstein, proyecto que trata, de acuerdo a Rhees, de clarificar el papel y funcionamiento del lenguaje en las actividades prácticas y las empresas intelectuales tanto desde un punto de vista lógico como desde un punto de vista discursivo. Enfáticamente Rhees (2003, p. 67) sostiene que el tema principal de OC se deja apreciar sólo si se admite que Wittgenstein discute con Moore respecto del papel y uso de "saber".

Esto me sugiere que OC de Wittgenstein, así como probablemente el Tractatus y las Investigaciones, es el producto de una respuesta, una reacción a lo que Wittgenstein consideró siempre falta de criterio en el entendimiento del lenguaje y el puzzle filosófico. Se trataría de un juego constante entre un proyecto filosófico con cierta coherencia temática y un hábito de respuesta a su contexto.

Lo anterior, sin embargo, no es lo más importante que cabe concluir en torno a los problemas que suscita la lectura de OC. Me parece que un punto interesante sería profundizar en la distinción "certeza objetiva" y "certeza subjetiva" -concepto de Stroll este último- o "certidumbres objetivas" -noción de Moyal-Sharrock-. En la distinción hay una tensión no totalmente explicada de cómo la pertenencia a grupo se solapa o cruza con la formación de actitudes y creencias individuales. Si la certeza objetiva (expresada en comportamientos, sea de sustrato biológico, como en el caso de la proposición bisagra "Tengo un cuerpo", ya de sustrato histórico como en el caso de la proposición bisagra "La tierra existe hace más de 100 años"), es parte de un marco de referencia heredado, común, que no obstante sólo se experimenta individualmente, es decir, fuera del espacio del agente no existen, entonces ¿cuál es el mecanismo de inserción y desarrollo de tales certezas?, ¿cómo se mantienen?, y ¿cómo se conocen y justifican? Según se sigue de la argumentación de Wittgenstein, a la primera pregunta se le respondería que es a partir de algún tipo de mecanismo evolutivo o, si se prefiere, de un cierto innatismo que la certeza objetiva se encapsula en la información de la especie; a la segunda pregunta se le respondería que la certeza objetiva se mantiene por su papel, literalmente, vital en una comunidad de habla; y a la tercera pregunta se le respondería que no la conocemos y está exenta de justificación. Sin embargo, como lo discute Pritchard (2007, pp. 205-207) a propósito de la lectura de Wright (2004) de OC, están exentas de justificación epistémica, pero sí tienen justificación pragmática vía las presuposiciones externas que suponen su formación. De modo que 
el problema del externalismo es una dimensión abierta a la discusión, a saber, el esclarecimiento de cómo se reconstruyen e interpretan las estructuras totales sobre las que avanzan las proposiciones bisagras.

El problema del anti-escepticismo que se le atribuye a Wittgenstein en OC es otro que requeriría mayor profundización. Si el escéptico es aquel que cree, o duda sistemáticamente, que no hay conocimiento último sobre hechos, entonces, en este sentido básico, Wittgenstein es un escéptico. Pero Wittgenstein lo es por razones diferentes. La posibilidad de duda es cuestión epistémica, y la certeza animal, aquélla sobre la que nuestra vida tiene sentido, no entra en el juego del escepticismo, no cabe como duda filosófica, pues dudar de ella, en una comunidad de habla, significaría que nos declaren desadaptados. Wittgenstein duda del posible conocimiento de la existencia de la certeza animal porque sobre ella no cabe duda alguna. Si dudo lo que afirma la proposición "Aquí tengo una mano", esta actitud no sería otra cosa que un pasatiempo heurístico, y no una duda real. Si el escéptico duda de este enunciado-hecho no sería una duda legítima, o por lo menos no más allá del escritorio del filósofo ${ }^{12}$. El escéptico es un dudoso obsesivo, a pasos de la neurosis.

En circunstancias normales "Tengo dos manos" constituye una proposición bisagra, pero bien puede ser el caso que pueda ser una proposición empírica y como tal sujeta a legítima duda, como cuando recién operado por un accidente la sostenga luego de que quiten el vendaje. Pero justamente circunstancias especiales como ésta hacen de la proposición bisagra estar fuera de duda en nuestro andar cotidiano. De todos modos, el estatus de la certeza como proposición bisagra debe apoyarse en criterios claros para distinguirlo de las manifestaciones empíricas que, con contenidos lingüísticos similares pero con actitudes intencionales distintas, parecieran confundir su análisis. Es un tema que también puede estar en una agenda de investigación futura.

"Quiero decir, pues, algo que me suena a pragmatismo" (OC $\$ 422)$ es, para ir cerrando estas ampliaciones, una afirmación de Wittgenstein un tanto difusa, ya que él rechazó cualquier posible identificación con esta corriente, porque nunca quiso que se confundiera "el significado es el uso" con "el significado es lo útil"13. Sin embargo, nuestras creencias fundacionales, nuestras proposiciones bisagras, la certeza animal, si ha de tener un

${ }^{12}$ Barry Stroud (2002) comienza su libro The Quest for Reality citando como epígrafe la siguiente afirmación de Wittgenstein: “Así es como deberían saludarse los filósofos: Tómate tu tiempo".

${ }^{13}$ Véase parágrafo 266 de Últimos escritos sobre filosofía de la psicología, Vol. 1. 
papel práctico, éste viene dado por el saber-cómo, por el hecho de que son formas de actuar. Como sostiene Brandom (2002), sólo en el sentido amplio de pragmatismo, aquel cuyo centro es la primacía de lo práctico, es que Wittgenstein cabría ser considerado como un pragmático. Pero hay que enfatizar: es el saber-cómo el que funciona como piso fundacional, nada más. Pero aunque no lo quisiera, el pragmatismo de Wittgenstein, así visto, es una cuerda que lo ata a su contexto. Sobre esto, que duda cabe, más habrá que investigar para comprender la filosofía de Wittgenstein y la de su tiempo.

\section{REFERENCIAS}

Brandom, R. (2002). "Pragmatics and Pragmatisms", en J. Conant y U. Zeglen (eds.) Hilary Putnam: Pragmatism and Realism. London: Routledge, 40-58.

Malcolm, N. (1986). Nothing is Hidden: Wittgenstein's Criticism of his early Thought, Oxford, Blackwell.

Moyal-Sharrock, D. (2004). "Introduction: The idea of a third Wittgenstein", en D. Moyal-Sharrock (ed.), The Third Wittgenstein: The Post-Investigations Works. Aldershot: Ashgate, 1-12.

. (2005). Understanding Wittgenstein's On Certainty. London: Palgrave. . (2007). "Unravelling Certainty" en D. Moyal-Sharrock y W. Brenner (eds.), Readings of Wittgenstein's On Certainty. London: Palgrave, 76-100.

Pritchard, D. (2007): "Wittgenstein's On Certainty and Contemporary Anti-scepticism", en D. Moyal-Sharrock y W. Brenner (eds.), Readings of Wittgenstein's On Certainty. London: Palgrave, 189-224.

. (forthcoming). "Wittgenstein on Scepticism", en M. McGinn (ed.), Oxford Handbook to Wittgenstein. New York: Oxford University Press.

Rhees, R. (2003). Wittgenstein's On Certainty. There- Like Our Life. Oxford: Blackwell.

Searle, J. (2001). Rationality in Action (Jean Nicod Lectures). Cambridge: The MIT Press.

Searle, J. (2002). "Conversation", en J. Searle, Consciousness and Language. Cambridge: Cambridge University Press, 180-202.

Soames, S. (2003). The age of meaning. Philosophical Analysis in the Twentieth Century. Princeton: Princeton University Press.

Stroll, A. (1994). Moore and Wittgenstein on Certainty. Oxford: Oxford University Press.

. (2007). "Why On Certainty matters", en D. Moyal-Sharrock y W. Brenner (eds.), Readings of Wittgenstein's On Certainty. London: Palgrave, 33-46.

Stroud, B. (2002). The Quest for Reality: Subjectivism and the Metaphysics of Colour. Cambridge: Oxford University Press. 
von Wright, G. H. (1982). Wittgenstein. Oxford: Basil Blackwell.

Williams, M. (2007). "Why Wittgenstein Isn't a Foundationalist”, en D. Moyal-

Sharrock y W. Brenner (eds.), Readings of Wittgenstein's On Certainty. London: Palgrave, 47-58.

Wittgenstein, L. (1980). Culture and Value. Oxford, Blackwell. . (1991). On certainty. Oxford, Blackwell. . (1999). Tractatus Logico-Philosophicus. Trad. Jacobo Muñoz. Madrid: Alianza.

. (2002). Investigaciones filosóficas. Trad. Alfonso García y Ulises Moulines. Barcelona: Crítica. . (2008). Últimos escritos sobre filosofía de la psicología. Vol. I, trad. Luis Valdés Villanueva. Madrid: Tecnos.

Wright, C. (2004). "Wittgensteinian Certainties", en D. McManus (ed.), Wittgenstein and Scepticism. London: Routledge, 22-55. 